\title{
Adaptive psychophysical procedures, loss functions, and entropy
}

\author{
Elena Kelareva and James Mewing \\ University of Melbourne, Melbourne, Victoria, Australia \\ Andrew Turpin \\ RMIT University, Melbourne, Victoria, Australia \\ AND \\ ANTHONY WiRIH \\ University of Melbourne, Melbourne, Victoria, Australia
}

\begin{abstract}
In this article, we present a new strategy for locating a point on a psychometric function (threshold determination) for yes-no procedures, called ENTFIRST. Our results show that it performs better than many existing strategies for estimating the $50 \%$ threshold that are commonly used in nonlaboratory settings. We also provide a review of existing algorithms for finding thresholds, with an emphasis on identifying the types of problems for which each algorithm is useful. Finally, we address a number of issues that are not adequately covered in the literature, including choosing an appropriate loss function to evaluate the performance of an algorithm for a given problem.
\end{abstract}

Efficiently locating single points on a psychometric function at some given detection probability is an important problem, particularly in clinical settings outside of psychophysics laboratories. For example, visual field tests rely on quickly finding the $T_{50}$ contrast detection threshold (i.e., the light intensity that can be detected $50 \%$ of the time) in many locations in the visual field using a yesno procedure (Henson, Chaudry, Artes, Faragher, \& Ansons, 2000). Similarly, methods for determining auditory thresholds are an active area of research (Eilers, Ozdamar, \& Steffens, 1993). Procedures that locate such thresholds repeatedly present the subject with stimuli of varying intensities and record whether the subject perceives each stimulus.

The probability of a person detecting a stimulus of a given intensity can be modeled by a psychometric function $F(x)$. This describes the probability that a particular subject will detect a stimulus of intensity $x$. One of the main problems in psychophysics is finding the threshold $T$ of a subject's psychometric function, where $T$ is commonly defined as the intensity value at which the stimulus is detected $50 \%$ of the time; that is, $T$ satisfies $F(T)=.5$ (Treutwein, 1995). The accuracy and the efficiency of the estimation of $T$ depend on the strategy used: the stimuli that are presented and the conclusions made from the responses to the stimuli. In an adaptive setting, the key question is how we choose the stimulus level for the next presentation on the basis of the responses that have already been given.
Before deciding on an estimation strategy, the researcher must first determine how the performance of the strategy (or the outcome of the strategy) will be measured. One principled way to do this is to choose an appropriate loss function. The best strategy will therefore minimize the expected loss, over all possible sequences of subject responses.

One major shortcoming of the existing psychophysics literature is the inconsistency in the choice of loss function used to evaluate different strategies. Different principles may be used to evaluate quite similar algorithms (King-Smith, Grigsby, Vingrys, Benes, \& Supowit, 1994; Watson \& Pelli, 1983). In this article, we investigate, for each of a number of applications, which loss function is most appropriate and compare the performance of a number of existing algorithms with respect to several different loss functions.

Many existing strategies use Bayesian techniques to determine the threshold estimate and stimulus query levels. The initial stimulus is chosen according to some function of the prior distribution of thresholds, often estimated from the population. This distribution is then updated to reflect the knowledge gained from the initial response. The response function, generally of the form $R(T, x)=$ $F(x-T)$, where $T$ is a location parameter, describes the probability that a person with true threshold $T$ detects a stimulus with intensity $x$. Since the prior distribution and response functions are themselves estimates, algorithms must be robust to inaccuracies in these distributions and

A.Wirth, awirth@unimelb.edu.au 
their parameters. In practice, there may be some observer bias due to a shift in the detection criterion over time, but we assume that this can be controlled. In this article, query selection strategies described by Watson and Pelli (1983), King-Smith et al. (1994), Kontsevich and Tyler (1999), and Alcalá-Quintana and García-Pérez (2004) are each evaluated using three different loss functions for a variety of priors and response functions.

Our main contribution is a new strategy for finding the threshold, ENTFirsT. We do this in the context of stimuli and thresholds in a discrete set, with a small number of queries and constant detection sensitivity throughout the experiment. This is the typical situation for clinical automated perimetry, in which contrast detection thresholds are measured in visual fields. We also assume that we are testing using a yes-no detection task, which is typically necessary in clinical settings when observers are untrained for the task. Finally, note that we do not directly address the issue of confidence limits for threshold estimation in this article.

The ENTFIRST strategy builds on the approaches used by King-Smith et al. (1994) and Kontsevich and Tyler (1999) to give a new, more general query selection method. The initial queries are chosen so as to minimize the expected entropy of the posterior distribution of thresholds, which is the approach used by Kontsevich and Tyler. However, the last few queries are chosen to minimize the expected loss of the posterior distribution. This modification was inspired by the success of King-Smith et al.'s approach in minimizing the expected variance of the posterior distribution, in which the loss function is the squared distance from the true threshold. The improvement made by our ENTFIRST scheme is an order of magnitude greater than the improvements made by previous strategies in the context that we are investigating.

\section{BACKGROUND}

Psychophysical procedures are used in many areas of medical diagnosis, particularly those that involve measuring a subject's performance in perception tasks. The most common clinical applications of psychophysics involve measuring defects in visual perception, such as visual field loss due to glaucoma (Henson et al., 2000; Turpin, McKendrick, Johnson, \& Vingrys, 2002a) and optic neuritis (Henson et al., 2000). Psychophysics has also been applied to measuring the perception of other senses, including sound (Ryan, 1976), taste, and smell (Linschoten, Harvey, Eller, \& Jafek, 2001).

\section{Classifying Psychophysical Procedures}

In his review of psychometric functions, Klein (2001) classified psychophysical procedures using three main distinguishing characteristics: detection versus discrimination questions, forced choice versus yes-no tasks, and constant stimuli versus adaptive methods. The forced choice versus yes-no distinction and the use of detection versus discrimination problems are both primarily associated with experimental design, rather than with the threshold estimation strategy. The merits of the first two distinc- tions are not investigated in detail here, since this article is focused on the algorithms used for query selection. The algorithms discussed in this article are principally applicable to yes-no methods; with minor modifications they could be applied to two-alternative forced choice methods (Klein, 2001), but we do not explore this here. We focus on adaptive methods, which assume that the results of previous queries can be taken into account when selecting the next stimulus level, and yes-no tasks. In general, one could aim to estimate the psychometric function $F(x)$, but traditionally, the focus has been on estimating the threshold, as it is in this article.

\section{Existing Algorithms: Bayesian}

Most adaptive psychophysical procedures are Bayesian in spirit; this article is focused on Bayesian strategies. The model starts with a prior distribution of thresholds, $P_{T}^{(0)}(T)$, perhaps determined from experience with the population. This distribution is used to select the first stimulus. The distribution of thresholds is updated to a posterior distribution according to the subject's response, by applying Bayes's Rule. This update procedure is repeated after each query so that

$$
P_{T}^{(i)}(t)= \begin{cases}P_{T}^{(i-1)}(t) R\left(t, q_{i}\right), & r_{i}=y e s, \\ P_{T}^{(i-1)}(t)\left[1-R\left(t, q_{i}\right)\right], & r_{i}=n o,\end{cases}
$$

where $P_{T}^{(i)}(t)$ is the probability of the true threshold being $t$ after $i$ queries, $q_{i}$ is the intensity of the $i$ th query, $r_{i}$ is the response to that query, and $R\left(t, q_{i}\right)$ is the probability of the answer $r_{i}$ to $q_{i}$ being yes if the true threshold $T$ is at intensity $t$. Note that the immediate consequence of Equation 1 is a set of values that do not sum to 1 , so the posterior distribution will have to be normalized if the absolute probability values are of interest. Typically only relative values are used, and so the normalizing step is omitted.

Bayesian methods are characterized by the query selection method, the stopping criterion, and the choice of final threshold estimate. Unfortunately, there is little consensus on how to evaluate estimation strategies, which leads to different heuristics being advocated (Alcalá-Quintana \& García-Pérez, 2004; Turpin et al., 2002a; Watson \& Pelli, 1983). Moreover, performance may vary depending on whether the evaluations were based on computer simulation (Watson \& Pelli, 1983) or on averaging the results of a number of laboratory experiments with human test subjects (Turpin et al., 2002a).

\section{Existing Algorithms: Alternatives}

Bootstrap or other resampling methods have been used to determine confidence limits in the estimation of thresholds (Hill, 2001; Wichmann \& Hill, 2001a, 2001b). This approach was extended to a Bayesian method by Kuss, Jäkel, and Wichmann (2005).

Alternatively, PEST, introduced by Taylor and Creelman (1967), repeatedly queries one point and uses the probability of detecting a stimulus at that intensity to estimate the distance from the threshold.

Some strategies exploit the similarity of threshold estimation to the search with errors problem. Although these 
strategies are adaptive, they do not fall under the Bayesian framework. One such algorithm is modified binary search (MOBS), proposed by Tyrell and Owens (1988), which selects queries using binary search, modified to allow for subject variability; it is used in a current commercial frequency doubling technology (FDT) perimetry machine (FDT Perimeter, Welch Allyn, New York). Turpin et al. (2002a) investigated the performance of a modified version of MOBS, called rapid efficient binary search (REBS).

\section{Adaptive Bayesian Psychophysical Procedures}

In this section, we explore in detail a variety of Bayesian adaptive threshold estimation schemes. The problem of choosing an appropriate loss function for a given application is addressed, and optimal final threshold estimates for each loss function are discussed. Six different approaches to query selection are investigated, including most of the existing Bayesian psychophysical methods. Features of the problem that may influence algorithm performanceincluding the prior distribution of thresholds, $P_{T}^{(0)}(T)$; the response function, $R(T, x)$; and the lapse and guess rates, $\lambda$ and $\gamma$, respectively - are also examined.

Bayesian schemes are classified primarily by their choice of queries, final estimate, and stopping criterion. The querying method is a function of the distribution of thresholds, $P_{T}^{(i)}(T)$, and the response function, $R(T, x)$. The final estimate is often - and is in our approach - a function of the posterior distribution of thresholds, $P_{T}^{(N)}(T)$, after the final response (to the $N$ th query) is known. In theory, the stopping rule for the process is a function of the distribution of thresholds, $P_{T}^{(i)}(T)$, and the number of queries made so far, $i$ (to determine the value of $N$ ). However, before we can determine the best query function, final estimate, and stopping criterion, a scheme for evaluating the performance of a psychophysical method must be decided on. The goal in the problem is to choose a sequence of queries that minimizes some loss function. For any given real-world problem, it is necessary to first choose an appropriate loss function and only then to select the adaptive scheme to minimize the expected loss.

\section{Choosing the Loss Function}

Existing literature offers a number of performance criteria for psychometric procedures. In general, we wish to estimate $F(x)$, but since the problem is often reduced to estimating the threshold $T$, the idea is to determine a penalty for misestimating the true threshold $T$. We refer to this penalty as the loss function and write $L(T, t)$ for the cost of choosing the estimate $t$ for the true threshold $T$. The aim is to minimize expected loss over all possible response patterns by the subject and over the prior distribution.

Turpin et al. (2002a) and King-Smith et al. (1994) actually considered two loss functions. The first is $t-T$, whose expected value is the bias of the procedure. The other is the loss function

$$
(t-T)^{2}-\text { Bias }^{2},
$$

whose expected value is, in fact, the variance of the error.
Kuss et al. (2005) considered the loss function $|t-T|$, the absolute distance from the true threshold, but focused on its median, rather than on its mean, as well as on confidence intervals for the threshold estimate. Watson and Pelli (1983) evaluated their QUEST procedure with the asymptotic efficiency measure proposed by Taylor and Creelman (1967): the product of the number of queries and the variance of the estimator of the threshold.

Treutwein (1995) presents an analysis of several performance measures, including bias, precision (inverse variance), and efficiency (the number of trials required to achieve a given precision). However, most of the individual studies cited by Treutwein provide very little information on why a particular performance measure should be chosen over any other. We now list various loss functions and seek to provide circumstances in which their use is justified.

Bias. If the estimation of the threshold will be carried out many times, a scheme with a low bias but high variance is unlikely to find the correct threshold on any one occasion, but averaging over many threshold estimates gives a result that is very close to the true threshold.

Absolute error. The ABsERR strategy minimizes the mean absolute distance, sometimes called the Manhattan, or $L_{1}$, distance. This is the average of the absolute distance $[L(T, t)=|T-t|]$ over all possible values of $T$. However, this loss function does not strongly penalize the strategy for having high error for a small proportion of the population. Therefore, ABSERR is suitable for situations in which it is more important to maximize average accuracy, rather than maximize the accuracy for the worst cases.

Square error. Here, we minimize the mean square distance, or variance, which we call SQERR. A scheme that minimizes the variance will heavily penalize any rare large errors, as opposed to more common but smaller errors. Therefore, the SQERR loss function is useful for situations in which a high error for a small proportion of subjects is unacceptable.

Categorical error. The mean categorical error, CATERR, is defined by the function

$$
L(T, t)= \begin{cases}0, & t=T \\ 1, & \text { otherwise }\end{cases}
$$

and is useful when the threshold must be determined exactly; any amount of error requires the subject to be retested. In this case, every result other than the correct one is equally undesirable.

Efficiency. Efficiency is the product of the standard error of the estimator of $T$ and the number of queries. This is useful when the desired precision is fixed and the number of questions varies. The previous performance measures do not account for the number of queries. Efficiency is an appropriate measure if each query only takes a short time or if it is vital to achieve some desired precision for each subject, even if that may require a large number of queries.

These performance measures and loss functions offer a suitably generic method of comparing estimation strategies. However, for some real-world tasks, more complex performance measures and loss functions may be required. 
Table 1

Expected Loss of Final Estimate Rules for Specific Loss Functions

\begin{tabular}{|c|c|c|c|c|c|c|c|c|c|}
\hline \multirow{3}{*}{$\begin{array}{l}\text { Query } \\
\text { Rule }\end{array}$} & \multicolumn{9}{|c|}{ Loss Function } \\
\hline & \multicolumn{3}{|c|}{$\begin{array}{c}\text { SQERR } \\
\text { Final Estimate }\end{array}$} & \multicolumn{3}{|c|}{$\begin{array}{c}\text { AвSERR } \\
\text { Final Estimate }\end{array}$} & \multicolumn{3}{|c|}{$\begin{array}{c}\text { CATERR } \\
\text { Final Estimate }\end{array}$} \\
\hline & Mean $^{\mathrm{a}}$ & Median & Mode & Mean & Median $^{b}$ & Mode & Mean & Median & Mode \\
\hline \multicolumn{10}{|c|}{$N=6$} \\
\hline MEAN & 0.876 & 0.879 & 0.888 & 0.653 & 0.651 & 0.652 & 0.551 & 0.547 & 0.544 \\
\hline MEDian & 0.893 & 0.896 & 0.916 & 0.665 & 0.661 & 0.663 & 0.561 & 0.555 & 0.549 \\
\hline MODE & 1.187 & 1.189 & 1.224 & 0.772 & 0.772 & 0.777 & 0.596 & 0.595 & 0.592 \\
\hline MinEnT & 0.880 & 0.888 & 0.921 & 0.669 & 0.660 & 0.666 & 0.573 & 0.556 & 0.549 \\
\hline \multicolumn{10}{|c|}{$N=9$} \\
\hline MEAN & 0.603 & 0.605 & 0.610 & 0.511 & 0.511 & 0.512 & 0.467 & 0.465 & 0.464 \\
\hline MEDIAN & 0.610 & 0.612 & 0.620 & 0.516 & 0.515 & 0.516 & 0.471 & 0.468 & 0.467 \\
\hline Mode & 0.720 & 0.724 & 0.734 & 0.572 & 0.571 & 0.573 & 0.502 & 0.499 & 0.499 \\
\hline MinEnT & 0.599 & 0.601 & 0.602 & 0.508 & 0.508 & 0.508 & 0.464 & 0.463 & 0.463 \\
\hline \multicolumn{10}{|c|}{$N=18$} \\
\hline MEAN & 0.319 & 0.319 & 0.319 & 0.310 & 0.310 & 0.310 & 0.306 & 0.306 & 0.306 \\
\hline MEDIAN & 0.320 & 0.320 & 0.320 & 0.311 & 0.311 & 0.311 & 0.307 & 0.307 & 0.307 \\
\hline MODE & 0.345 & 0.345 & 0.345 & 0.332 & 0.332 & 0.332 & 0.325 & 0.325 & 0.325 \\
\hline MinEnT & 0.314 & 0.314 & 0.314 & 0.307 & 0.307 & 0.307 & 0.303 & 0.303 & 0.303 \\
\hline
\end{tabular}

aWhen the loss is the expected square distance from the true threshold, the optimum final estimate is the mean (in every example tested). bFor the expected absolute distance, the best final estimate is the median. 'In the categorical error case, the best final estimate is the mode.

For example, it may be known that the subjects presenting for testing will mostly have thresholds within a given range or that subjects with certain threshold values may be particularly difficult to test; thus, the algorithm should be optimized to give good results for that range of thresholds. Choosing an appropriate loss function to reflect any such constraints is necessary in order to accurately evaluate performance of different schemes for the problem at hand. However, as is shown in Table 1, the choices of loss function and final threshold estimate are intimately related.

\section{Query Selection}

Once a loss function for the given problem has been selected, attention turns to the adaptive scheme itself. Bayesian query selection methods that have been documented in the literature include querying the mean (KingSmith et al., 1994), median, and mode (Watson \& Pelli, 1983) of the current distribution of thresholds. We refer to these as the MEAN, MEDIAN, and MODE schemes, respectively. Another well-known scheme involves selecting the stimulus that minimizes the expected entropy (MINENT; Kontsevich \& Tyler, 1999) or the variance (MINVAR; King-Smith et al., 1994) of the posterior distribution of thresholds. Recall that the entropy, $H$, of a probability distribution $P(x)$ over the range $\left\{x_{1}, \ldots, x_{K}\right\}$ is defined as

$$
H(P(x))=-\sum_{i=1}^{K} P\left(x_{i}\right) \log _{2} P\left(x_{i}\right) .
$$

Query selection schemes based on lookahead give rise to another question. How many steps is it best to look ahead? In general, lookahead methods minimize the expected variance or entropy of the posterior distribution after only one query. However, two-step lookahead can be used instead to minimize these quantities for the posterior distribution after two queries. King-Smith et al. (1994) showed that the extra computation time required to look ahead more than one step is not compensated for by a significant improvement in scores. Although a valid conclusion in 1994, current computing power allows two- and three-step lookahead to be calculated efficiently, and it is a topic worth revisiting.

We extend the work of Alcalá-Quintana and GarcíaPérez (2004), providing a comprehensive evaluation of query selection methods for the various loss functions. The query functions investigated in this article include all of the functions described above, as well as a MinLoss function, which minimizes the expected loss of the posterior distribution. For the SQERR loss function, MinLoss is equivalent to MINVAR; we also investigate the behavior of MinLoss with the ABSERR and CATERR loss functions. The MinEnT, MinVAR, and MinLoss functions are investigated with both one- and two-step lookahead.

\section{Final Threshold Estimate}

The final estimate for the location of the threshold should minimize the expected value of the loss function over the posterior distribution of thresholds, $P_{T}^{(N)}(T)$, after $N$ queries. For the three loss functions used to evaluate estimating strategies in this article-SQERR, ABSERR, and CATERR - the respective point estimates that minimize these loss functions are the mean, median, and mode of the posterior distribution. This is a standard result that is found in many statistics textbooks, such as that by Hastie, Tibshirani, and Friedman (2001).

It is not surprising, therefore, that Alcalá-Quintana and García-Pérez (2004) found that using the mean of the final posterior distribution as the estimate gave the best results. They used a square distance loss function as their performance measure.

The preliminary results in the Simulations section confirm this nexus between final estimate methods and loss 
functions. Therefore, for the remainder of this article, whenever a scheme is evaluated using one of the three loss functions above, the final estimate will always be selected to be the one that minimizes the expected value of that particular loss function.

\section{Number of Queries}

The existing literature presents a number of different stopping criteria for adaptive threshold estimation. One rule for choosing the number of questions is to continue asking until a sufficiently high accuracy is reachedthat is, when the confidence interval of the threshold becomes sufficiently narrow (Watson \& Pelli, 1983). Another, more popular, option is to simply stop after some specified number of queries, which is the approach used by Kuss et al. (2005), Kontsevich and Tyler (1999), and many others.

The stopping rule depends on the real-world application of the psychophysical procedure. In a clinical situation, the number of queries is generally restricted to no more than 8-10 (Bengtsson \& Heijl, 1998), whereas in a laboratory setting, the test subjects may be asked hundreds of questions. The sheer number of queries that can be made in laboratory psychophysics means that even nonoptimal strategies will produce accurate results. Therefore, in this article we focus our analysis on schemes to be used in a clinical setting where producing accurate results with limited available time is important. As a result, we deal only with stopping rules that have a small fixed number of queries $(6,9$, or 18$)$.

\section{Other Features of the Problem}

For estimating the threshold, a Bayesian adaptive procedure attempts to model a real-life situation. The loss function needs to be chosen carefully to reflect the realworld priorities of the experiment; the prior distribution and the response functions should be accurate representations of the subject being tested. For example, the prior distribution may vary according to trends in the age and health of the subjects: $P_{T}^{(0)}(T)$ for the general population may be different from those presenting for a vision test. In addition, good psychometric functions tend to include the lapse and guess rates, $\lambda$ and $\gamma$, which represent the probability of a subject accidentally ignoring the stimulus or guessing a correct answer.

Although $R(T, x), \lambda$, and $\gamma$ are generally assumed to be the same for all subjects (Alcalá-Quintana \& GarcíaPérez, 2004; Klein, 2001; Kuss et al., 2005), they may also vary because of the subjects' attentiveness and other individual characteristics. The assumption that the response behavior is the same for all subjects (save for the location parameter) is made because allowing for extra variability in the response function makes estimating the threshold an overly difficult problem. Besides, the existing algorithms produce sufficiently good results despite the assumption that $R(T, x)$ is the same for all subjects. In this article, we will follow the approach of other studies in the field in assuming that $R(T, x), \lambda$, and $\gamma$ are the same for all subjects, unless stated otherwise. However, an algorithm must be robust enough to deal with uncertainty in $P_{T}^{(0)}(T)$ and $R(T, x)$. The optimal choice of prior distribution and response function in situations in which one or both of these are not known with sufficient accuracy must also be considered.

\section{SIMULATIONS}

\section{General Method}

Our aim in this study was to investigate the statistical underpinnings of the models for psychometric functions and their estimation. Therefore, computer simulations were the source of the experimental results, rather than testing of human subjects, which seems a sensible first step. Having said that, a simulation is only as useful as its underlying assumptions, but we are not in a position to evaluate the assumptions, merely their consequences.

In all but one of the simulations, it was possible to make an exact calculation of the expected loss of the threshold estimate in a given scenario. The term scenario refers to the (true) prior distribution of thresholds, the response function, and the estimation tools (the query and final estimate policies). In this case, assuming that $N$ queries were to be made,

$$
E[\text { Loss }]=\sum_{\left(r_{i}\right) \in\{y e s, n o\}^{N}} P\left(r_{1}, \ldots, r_{n}\right) \sum_{t} P_{T}^{(0)}(t) L(T, g),
$$

where $P\left(r_{1}, \ldots, r_{n}\right)$ is the probability of the sequence of responses and $g$ is the final threshold guess. Note that for a specific sequence of responses, it is possible to determine from the query policy and the final estimate policy the stimulus levels presented and, thus, the probability of the sequence of responses. The final sum is over the various possibilities for the true threshold $T$. There are roughly $2^{N}$ different terms to calculate in this sum, one for each sequence of responses. If 18 queries are made, this calculation becomes large but reasonable on today's computers. Adding lookahead to the query selection process, however, only lengthens the calculation time. Despite this, with the exception of the final table, no statements about significance are necessary, since the calculations are exact, not estimates. Unless specified otherwise, somewhat inspired by the visual field application, we assumed that the threshold and the stimulus values were drawn from the discrete space of size 10.

\section{Confirming the Nexus Between Loss and Final Estimate Functions}

\section{Method}

As was mentioned previously, there is an obvious theoretical connection between the loss functions and the final estimate rules. For example, the mean of a distribution is the best estimator for minimizing SQERR. We evaluated this in practice by selecting a range of final estimate policies, with the prior threshold population following the uniform distribution. The maximum number of queries was 6,9 , or 18. These results were obtained by exact evaluation with a uniform prior threshold distribution, the logistic response distribution with scale parameter $s=1.0$ (and thus variance $=\pi^{2} / 3$ ). This will be the standard setup for all of the simulations unless otherwise stated.

\section{Results}

We observe that, without exception, the statistical theory, relating loss function and threshold estimate rule, referred to in the Final Threshold Estimate section, is borne out by the results in Table 1. We therefore, in the remainder of this article, restrict attention to the canonical final estimate rule for each loss function. We also note that in most cases, the MINENT query scheme for the stimulus is best, although for short query sequences, the MEAN is superior. This observation holds, regardless of the choice of loss function and accompanying final estimate method. 
Table 2

Expected Loss for Each Combination of Loss Function and Number of Queries

\begin{tabular}{|c|c|c|c|c|c|c|c|c|c|}
\hline \multirow{3}{*}{$\begin{array}{c}\text { Query } \\
\text { Rule }\end{array}$} & \multicolumn{9}{|c|}{ Loss Function and Final Estimate } \\
\hline & \multicolumn{3}{|c|}{ SQERr Mean } & \multicolumn{3}{|c|}{ AвsErk Median } & \multicolumn{3}{|c|}{ CatErk Mode } \\
\hline & $N=6$ & $N=9$ & $N=18$ & $N=6$ & $N=9$ & $N=18$ & $N=6$ & $N=9$ & $N=18$ \\
\hline MEAN & 0.876 & 0.603 & 0.319 & $0.651^{\dagger}$ & 0.511 & 0.310 & $0.544^{\dagger}$ & 0.464 & 0.306 \\
\hline MEDIAN & 0.893 & 0.601 & 0.320 & 0.661 & 0.515 & 0.311 & 0.549 & 0.467 & 0.307 \\
\hline MODE & 1.187 & 0.720 & 0.345 & 0.772 & 0.571 & 0.332 & 0.592 & 0.499 & 0.325 \\
\hline MinEnT-1 & 0.880 & 0.599 & 0.314 & 0.660 & 0.508 & 0.307 & 0.549 & 0.463 & 0.303 \\
\hline MinEnt-2 & 0.880 & $0.597^{\dagger}$ & 0.314 & 0.656 & $0.507^{\dagger}$ & 0.307 & 0.546 & $0.462^{\dagger}$ & 0.303 \\
\hline MINVAR-1 & $0.867^{\dagger}$ & 0.600 & $0.312^{\dagger}$ & 0.659 & 0.509 & $0.304^{\dagger}$ & 0.551 & 0.464 & 0.301 \\
\hline MINVAR-2 & 0.873 & 0.601 & $0.312^{\dagger}$ & 0.662 & 0.510 & $0.304^{\dagger}$ & 0.552 & 0.464 & 0.301 \\
\hline
\end{tabular}

$\dagger$ The least observed average error.

\section{Lookahead and the Minimization of Entropy}

\section{Method}

Lookahead was introduced as a way of improving the quality of queries. King-Smith et al. (1994) were somewhat restricted by the computational power at the time and concluded that two-step lookahead did not provide much advantage over the usual one-step. We repeat their experiments using one- and two-step lookahead for the MinEnT and MinVar procedures, labeled MinEnt-1, MinEnT-2, MINVAR-1, and MINVAR-2, respectively, with the results shown in Table 2.

\section{Results}

It is hard to make any firm conclusions from the results in Table 2, except perhaps that choosing the MoDE of the current distribution for the query intensity is a poor strategy. In general, the extra lookahead step does not decrease the expected loss by much and, in some cases, increases the expected loss.

\section{Blending Entropy and the Loss Function: Our ENTFiRst Algorithm}

\section{Method}

Theoretical analyses, such as those of Paninski (2005), confirm that, asymptotically, the optimal query selection strategy is to maximize the mutual information between the distribution of $T$ and the distribution of the $\left\{q_{i}, r_{i}\right\}$ pair for query $i$. This is essentially the MinEnT strategy. In practice, however, we are not interested in the asymptotic performance but in how to minimize the error in the estimation of the threshold after a relatively small number of queries. We therefore use the following approach, which we call ENTFIRST, assuming that $N$ queries will be made: Queries 1 to $N-k$ follow the MinEnT strategy; the last $k$ queries follow the MinLoss strategy.

There are two parameters to be set for ENTFIRST: the value of $k$, and the number of steps of lookahead relied on by MinENT and MinLoss. Table 3 shows a comparison between versions of ENTFIRST with various parameter choices. Note that ENTFIRST with $k=0$ is merely the MINENT algorithm. Henceforth, a specific ENTFIRST scheme will be referred to with its parameters as ENTFIRST-lookahead- $k$. In this and all subsequent simulations, unless otherwise noted, the number of queries, $N$, is 9 .

\section{Results}

On the one hand, it seems from Table 3 that there is little to be gained from two steps of lookahead, rather than one. On the other hand, the ENTFIRST algorithm appears to produce a lower average loss than MinENT $(k=0)$, even with only one final step of MinLoss - that is, $k=1$.

\section{Robustness to Prior and Response Distributions}

\section{Method}

In order to establish ENTFIRST as a suitable, robust alternative threshold estimation strategy, we investigated how it performed with different distributions and with multiple lapse and guess rates. We first varied the prior and response distributions. The three prior distributions were the uniform distribution (as used in the simulations above); the normal distribution, with $M=4.5$ and $S D=2.5$; and an asymmetric distribution, roughly approximating the bimodal distribution of contrast detection thresholds in subjects with and without glaucoma (Vingrys \& Pianta, 1999). We considered the effect of one prior distribution generating the observed data and another prior distribution being used by the query generator and threshold estimator. Note that we assume that the 10 stimulus values are $0-9$. In addition to the usual logistic response curve, we tested a cumulative normal response with $S D=10 / 3$. Again, we considered the effect of one response curve generating the data, with another being assumed by the estimation algorithm. The loss function was SQERR, with the mean of the final distribution used as the threshold estimate. The losses for each combination of parameters were determined exactly.

\section{Results}

In Table 4, we see that the ENTFIRST query rule achieved a lower average loss than all the other schemes on every combination of prior distribution and response function. In addition, if we consider the improvement in loss that results from a new scheme to be

$$
1-\frac{\operatorname{loss}(\text { new })}{\operatorname{loss}(\text { old })}
$$

Table 3

Evaluating the Effect of the Amount of Lookahead and Number of MinEnT Steps

\begin{tabular}{cccc}
\hline \multirow{4}{*}{$\begin{array}{c}\text { No. } \\
\text { Steps }\end{array}$} & \multicolumn{3}{c}{ Loss Function and Final Estimate } \\
\cline { 2 - 4 } & SQERR Mean & ABsERR Median & CATERR Mode \\
\hline & \multicolumn{3}{c}{ Lookahead 1 } \\
1 & 0.603 & 0.500 & 0.469 \\
2 & $0.564^{\dagger}$ & 0.489 & 0.451 \\
3 & 0.566 & 0.491 & 0.452 \\
& 0.567 & 0.491 & 0.452 \\
0 & & Lookahead 2 & \\
1 & 0.601 & 0.500 & 0.460 \\
2 & 0.565 & $0.488^{\dagger}$ & $0.450^{\dagger}$ \\
3 & 0.566 & 0.490 & 0.451 \\
\end{tabular}

$\uparrow$ The least observed average error. 
Table 4

Expected Loss of Query Rules for Different Combinations of Prior and Response Functions

\begin{tabular}{|c|c|c|c|c|c|c|}
\hline \multirow[b]{3}{*}{ Query Rule } & \multicolumn{6}{|c|}{ Prior } \\
\hline & \multicolumn{2}{|c|}{$\begin{array}{l}\text { UNIFORM } \\
\text { Response }\end{array}$} & \multicolumn{2}{|c|}{$\begin{array}{l}\text { NORMAL } \\
\text { Response }\end{array}$} & \multicolumn{2}{|c|}{$\begin{array}{l}\text { BIMODAL } \\
\text { Response }\end{array}$} \\
\hline & LOGISTIC & CNORMAL & LOGISTIC & CNORMAL & LOGISTIC & CNORMAL \\
\hline MEAN & 0.603 & 1.404 & 0.605 & 1.334 & 0.240 & 0.864 \\
\hline MEDIAN & 0.610 & 1.416 & 0.605 & 1.338 & 0.357 & 0.998 \\
\hline MODE & 0.720 & 1.581 & 0.606 & 1.338 & 0.437 & 1.006 \\
\hline MINENT-1 & 0.599 & 1.398 & 0.601 & 1.333 & 0.161 & 0.752 \\
\hline ENTFIRST-1-1 & $0.564^{\dagger}$ & $1.349^{\dagger}$ & $0.568^{\dagger}$ & $1.282^{\dagger}$ & $0.158^{\dagger}$ & $0.743^{\dagger}$ \\
\hline
\end{tabular}

Note-The square loss function and mean final estimate were used. tThe least observed average loss.

Table 5

Evaluation of Robustness of Query Rules to Misunderstanding of Prior Rule

\begin{tabular}{|c|c|c|c|c|c|c|c|c|c|}
\hline \multirow[b]{3}{*}{ Query Rule } & \multicolumn{9}{|c|}{ True Prior } \\
\hline & \multicolumn{3}{|c|}{$\begin{array}{c}\text { UNIFORM } \\
\text { Assumed Prior }\end{array}$} & \multicolumn{3}{|c|}{$\begin{array}{c}\text { NoRMAL } \\
\text { Assumed Prior }\end{array}$} & \multicolumn{3}{|c|}{$\begin{array}{c}\text { BIMODAL } \\
\text { Assumed Prior }\end{array}$} \\
\hline & UNIFORM & NORMAL & BIMODAL & UNIFORM & NORMAL & BIMODAL & UNIFORM & NORMAL & BIMODAL \\
\hline MEAN & 0.603 & 0.644 & 1.453 & 0.648 & 0.605 & 1.305 & 0.663 & 0.598 & 0.240 \\
\hline MEDIAN & 0.610 & 0.644 & 1.474 & 0.653 & 0.605 & 1.316 & 0.683 & 0.581 & 0.357 \\
\hline MODE & 0.720 & 0.651 & 1.405 & 0.774 & 0.606 & $1.209^{\dagger}$ & 0.814 & 0.571 & 0.437 \\
\hline MINENT-1 & 0.599 & 0.633 & 1.348 & 0.639 & 0.601 & 1.245 & 0.658 & 0.580 & 0.161 \\
\hline ENTFIRST-1-1 & $0.564^{\dagger}$ & $0.599^{\dagger}$ & $1.304^{\dagger}$ & $0.597^{\dagger}$ & $0.568^{\dagger}$ & 1.237 & $0.620^{\dagger}$ & $0.559^{\dagger}$ & $0.158^{\dagger}$ \\
\hline
\end{tabular}

Note-A simulation was run for each combination of true and assumed prior. †The least observed average loss.

the improvement from MinEnT to ENTFiRST is at least eight times that from MEAN to MinEnt for the uniform and normal prior distributions. King-Smith et al. (1994) showed that MEAN is a sensible benchmark for the context that we are considering (discrete space, yes-no detection, pure threshold estimation). Of course, in practice, the true prior distribution and response functions may not be known: Estimates of them will be used to guide the query selection schemes. To model this situation, we preserved the logistic response function and tested the performance of the various methods when they assumed a different prior distribution from the true prior distribution of the population. Although somewhat idealized, this simulation does give some indication of algorithm robustness.

In the next simulation, we investigated the behavior of the algorithms when there was a misunderstanding of the prior distribution of thresholds. As is shown in Table 5, ENTFIRST was the most robust algorithm in every case except one: Here, the MODE query function, which performs worst when the assumed prior distribution is actually the true prior distribution, won. Again, the improvement made by ENTFIRST over previous schemes is, in several cases, substantially better than the improvement that MinEnT achieved.

In our next simulation, we fixed the prior distribution and assumed that it was known but tested how robust the query selection schemes were to a misunderstanding of the subject's response function. Table 6 shows that the ENTFIRST scheme was once again the best-performing strategy; its average loss is lower than any of the existing schemes, sometimes substantially so.

\section{Lapses and Guesses}

\section{Method}

It is not realistic to assume that the subject will respond to every stimulus or will respond honestly. For this reason, psychometric functions often include compensation for lapsing and guessing. Typically, we assume that all subjects have a common rate of lapsing $(\lambda)$ or guessing $(\gamma)$; this is an approximation, but it is still possible to gauge how robust the query strategies are. The logistic response distribution is modified with a shift and scale so that the response function has the required $\lambda$ and $\gamma$ values.

\section{Results}

Table 7 confirms that, even with a variety of lapse and guess rates, the ENTFIRST query selectors perform best. Admittedly, the improvement that ENTFIRST makes, compared with existing strategies, becomes less substantial as the lapse rate increases, but higher accuracy of the ENTFIRST strategy persists.

\section{Table 6}

Evaluation of Robustness of Query Rules to Misunderstanding of Response Function

\begin{tabular}{lccccc}
\hline & \multicolumn{3}{c}{ True Response Function } \\
\cline { 2 - 3 } & \multicolumn{2}{c}{$\begin{array}{c}\text { LoGISTIC } \\
\text { Assumed Response }\end{array}$} & & \multicolumn{2}{c}{ CNORMAL } \\
\cline { 2 - 3 } \cline { 5 - 6 } Query Rule & Assumed Response \\
\cline { 2 - 3 } \cline { 5 - 6 } MEAN & 0.603 & 0.649 & & 1.526 & 1.404 \\
MEDIAN & 0.610 & 0.655 & & 1.528 & 1.416 \\
MODE & 0.720 & 0.821 & & 1.720 & 1.581 \\
MiNENT-1 & 0.599 & 0.670 & & 1.498 & 1.398 \\
ENTFIRST-1-1 & $0.564^{\dagger}$ & $0.637^{\dagger}$ & & $1.412^{\dagger}$ & $1.349^{\dagger}$ \\
\hline
\end{tabular}

Note-A simulation was run for each combination of true and assumed response. The least observed average loss. 
Table 7

Expected Loss of Query Strategies With Typical Lapse and Guess Rates

\begin{tabular}{|c|c|c|c|c|c|c|c|c|c|}
\hline \multirow[b]{2}{*}{ Query Rule } & \multicolumn{3}{|c|}{$\begin{array}{l}\text { Lapse Rate }(\lambda)=0, \\
\quad \text { Guess Rate }(\gamma)\end{array}$} & \multicolumn{3}{|c|}{$\begin{array}{l}\text { Lapse Rate }(\lambda)=.05 \\
\text { Guess Rate }(\gamma)\end{array}$} & \multicolumn{3}{|c|}{$\begin{array}{l}\text { Lapse Rate }(\lambda)=.10, \\
\quad \text { Guess Rate }(\gamma)\end{array}$} \\
\hline & 0 & .05 & .10 & 0 & .05 & .10 & 0 & .05 & .10 \\
\hline MEAN & 0.603 & 0.693 & 0.787 & 0.700 & 0.823 & 0.956 & 0.798 & 0.955 & 1.126 \\
\hline MEDIAN & 0.610 & 0.718 & 0.834 & 0.709 & 0.840 & 1.003 & 0.827 & 0.985 & 1.189 \\
\hline Mode & 0.720 & 0.943 & 1.248 & 0.944 & 1.226 & 1.546 & 1.241 & 1.539 & 1.933 \\
\hline MinEnT-1 & 0.599 & 0.678 & 0.753 & 0.678 & 0.800 & 0.929 & 0.753 & 0.929 & 1.105 \\
\hline MinENT-2 & 0.597 & 0.677 & 0.753 & 0.678 & 0.800 & 0.931 & 0.754 & 0.929 & 1.107 \\
\hline ENTFIRST-1-1 & $0.564 \dagger$ & 0.647 & $0.727^{\dagger}$ & 0.647 & $0.768^{\dagger}$ & $0.899^{\dagger}$ & $0.727^{\dagger}$ & $0.899^{\dagger}$ & $1.078^{\dagger}$ \\
\hline ENTFIRST-2-1 & 0.565 & $0.646^{\dagger}$ & 0.731 & $0.646^{\dagger}$ & $0.768^{\dagger}$ & 0.902 & 0.728 & 0.900 & 1.079 \\
\hline
\end{tabular}

$\uparrow$ The least observed average loss.

Of course, just as the assumed response and prior distributions may not match the true distributions, the assumed lapse and guess rates may also be incorrect. We therefore tested the performance of the query strategies with various combinations of assumed and true lapse rates.

Even when the assumed and the true lapse and guess rates did not match, the ENTFIRST algorithm performed best, as Tables 8 and 9 show. Not only that, the improvement made by ENTFirst over MinEnT was at least half that made by MinEnT over choosing the MEAN of the distribution as the query point, except for one instance.

\section{Head-to-Head Comparisons}

Thus far, we have compared a number of algorithms for threshold estimation. Although the simulations in this article have followed standard procedure, we believe that we can make more powerful statistical statements.

\begin{abstract}
Method
A natural method of comparing two estimation techniques is to run each of them on the same subject and then to compare the two estimates obtained for closeness to the true threshold. Naturally, this simulation would need to be repeated with many subjects to obtain reliable results. For each pair of algorithms, we generated 10,000 "subjects" and ran the two algorithms in an attempt to estimate the threshold. We recorded the square of the error that each method incurred and, consequently, the difference in square errors. That is, for subject $i$, we have a value $Z_{i}=Y_{i}^{2}-X_{i}^{2}$, where $Y_{i}$ is the estimation error incurred by one method and $X_{i}$ is that incurred by the other. Wilcoxon's (1945) signed-rank test can then be used with these $Z_{i}$ values to determine the probability of obtaining the data observed under the null hypothesis: that the two estimation procedures are equally accurate at estimating the threshold. It is well known that for very large samples, the distribution of the statistic used in this test is close to the Gaussian distribution, which means that we can easily assign a significance value ( $p$ value) to the observations. This nonparametric test is effective because it assumes very little about the distribution of the errors made by the estimation algorithms, and there is no need to calculate confidence intervals.
\end{abstract}

Table 8

Testing the Robustness of the Query Rules to Misunderstandings of the Lapse Rate

\begin{tabular}{|c|c|c|c|c|c|c|c|c|c|}
\hline \multirow[b]{2}{*}{ Query Rule } & \multicolumn{3}{|c|}{$\begin{array}{l}\text { True Lapse Rate }(\lambda)=0, \\
\text { Assumed Lapse Rate }(\lambda)\end{array}$} & \multicolumn{3}{|c|}{$\begin{array}{l}\text { True Lapse Rate }(\lambda)=.05 \text {, } \\
\text { Assumed Lapse Rate }(\lambda)\end{array}$} & \multicolumn{3}{|c|}{$\begin{array}{c}\text { True Lapse Rate }(\lambda)=.10, \\
\text { Assumed Lapse Rate }(\lambda)\end{array}$} \\
\hline & .00 & .05 & .10 & .00 & .05 & .10 & .00 & .05 & .10 \\
\hline MEAN & 0.603 & 0.627 & 0.682 & 0.762 & 0.700 & 0.708 & 1.064 & 0.876 & 0.798 \\
\hline Median & 0.610 & 0.630 & 0.698 & 0.746 & 0.709 & 0.725 & 1.001 & 0.888 & 0.827 \\
\hline MoDE & 0.720 & 0.772 & 0.896 & 1.144 & 0.944 & 0.984 & 1.852 & 1.356 & 1.241 \\
\hline MinEnT-1 & 0.599 & 0.615 & 0.651 & 0.736 & 0.678 & 0.682 & 0.984 & 0.807 & 0.753 \\
\hline MinEnT-2 & 0.597 & 0.616 & 0.655 & 0.723 & 0.678 & 0.685 & 0.950 & 0.805 & 0.754 \\
\hline ENTFIRST-1-1 & $0.564^{\dagger}$ & $0.589^{\dagger}$ & $0.640^{\dagger}$ & 0.700 & 0.647 & $0.664^{\dagger}$ & 0.944 & 0.768 & $0.727^{\dagger}$ \\
\hline ENTFIRST-2-1 & 0.565 & $0.589^{\dagger}$ & 0.642 & $0.687^{\dagger}$ & $0.646^{\dagger}$ & 0.665 & $0.905^{\dagger}$ & $0.765^{\dagger}$ & 0.728 \\
\hline
\end{tabular}

Note-Guess rate $(\gamma)$ is 0 . †The least observed average loss.

Table 9

Testing the Robustness of the Query Rules to Misunderstandings of the Guess Rate

\begin{tabular}{|c|c|c|c|c|c|c|c|c|c|}
\hline \multirow[b]{2}{*}{ Query Rule } & \multicolumn{3}{|c|}{$\begin{array}{l}\text { True Guess Rate }(\gamma)=0, \\
\text { Assumed Guess Rate }(\gamma)\end{array}$} & \multicolumn{3}{|c|}{$\begin{array}{c}\text { True Guess Rate }(\gamma)=.05 \text {, } \\
\text { Assumed Guess Rate }(\gamma)\end{array}$} & \multicolumn{3}{|c|}{$\begin{array}{c}\text { True Guess Rate }(\gamma)=.10 \\
\text { Assumed Guess Rate }(\gamma)\end{array}$} \\
\hline & .00 & .05 & .10 & .00 & .05 & .10 & .00 & .05 & .10 \\
\hline Mean & 0.603 & 0.624 & 0.676 & 0.740 & 0.693 & 0.702 & 0.987 & 0.844 & 0.787 \\
\hline MEDIAN & 0.610 & 0.635 & 0.703 & 0.775 & 0.718 & 0.730 & 1.091 & 0.916 & 0.834 \\
\hline MODE & 0.720 & 0.772 & 0.898 & 1.209 & 0.943 & 0.984 & 2.086 & 1.370 & 1.248 \\
\hline MinEnT-1 & 0.599 & 0.615 & 0.651 & 0.732 & 0.678 & 0.682 & 0.969 & 0.807 & 0.753 \\
\hline MinENT-2 & 0.597 & 0.614 & 0.652 & 0.753 & 0.677 & 0.680 & 1.042 & 0.806 & 0.753 \\
\hline ENTFIRST-1-1 & $0.564 \dagger$ & 0.589 & 0.640 & $0.691^{\dagger}$ & 0.647 & 0.664 & $0.917^{\dagger}$ & 0.768 & $0.727^{\dagger}$ \\
\hline ENTFIRST-2-1 & 0.565 & $0.588^{\dagger}$ & $0.640^{\dagger}$ & 0.714 & $0.646^{\dagger}$ & $0.663^{\dagger}$ & 0.993 & $0.766^{\dagger}$ & 0.731 \\
\hline
\end{tabular}

Note-Lapse rate $(\lambda)$ is 0 . †The least observed average loss. 
Table 10

Wilcoxon $p$ Values for Head-to-Head Comparisons Between Query Rules

\begin{tabular}{|c|c|c|c|c|c|c|c|}
\hline \multirow{2}{*}{$\begin{array}{c}\text { Inferior } \\
\text { Query Rule }\end{array}$} & \multicolumn{7}{|c|}{ Query Rule With Superior Estimation } \\
\hline & MEAN & MEDIAN & MoDE & MinENT-1 & MinEnT-2 & ENTFIRST-1-1 & ENTFIRST-2-1 \\
\hline MEAN & & & & .116 & & .00707 & .103 \\
\hline MEDIAN & .139 & & & .00919 & .155 & $.000279^{*}$ & $8.44 \mathrm{E}-08^{*}$ \\
\hline MoDe & $5.96 \mathrm{E}-11^{*}$ & $6.4 \mathrm{E}-09^{*}$ & & $5.75 \mathrm{E}-13^{*}$ & $1.67 \mathrm{E}-15^{*}$ & $<1 \mathrm{E}-15^{*}$ & $<1 \mathrm{E}-15^{*}$ \\
\hline MinEnT-1 & & & & & & $.000334^{*}$ & $.000598^{*}$ \\
\hline MinENT-2 & .0463 & & & .456 & & .003 & $3.38 \mathrm{E}-07^{*}$ \\
\hline ENTFIRST-1-1 & & & & & & & \\
\hline ENTFIRST-2-1 & & & & & & .456 & \\
\hline
\end{tabular}

*With so many pairwise comparisons, only a $p$ value less than .002 should be considered significant.

\section{Results}

Table 10 shows the outcome of each head-to-head comparison in which a uniform prior distribution and a logistic response curve were used. For each pair of algorithms, there is a $p$ value, indicating to what extent we believe that one method is clearly superior to the other. In addition, to make it clearer which of the two methods obtained better threshold estimates, we have recorded the $p$ value in the column of the algorithm that obtained more accurate results.

Our main aim in Table 10, and indeed in the study as a whole, is to compare ENTFIRST with the other query schemes. There is ample evidence to reject the ModE method, because its least-significant defeat has a $p$ value of less than $10^{-8}$. We cannot conclude that two steps of lookahead are better than one step (for either the ENTFIRST or the MinEnT scheme). The MEDIAN method is only beaten significantly by the new ENTFIRST schemes. The key comparisons, naturally, are between ENTFIRST and MEAN and between ENTFIRST and MinEnT. On these comparisons, the MEAn fares better, not obviously defeated by any other method, whereas there is strong evidence that the ENTFIRST schemes are superior to the MinEnT schemes. Note that we used one-sided tests and $p$ values because our alternative hypotheses could be expressed as a variation from the null hypothesis in a particular direction.

\section{CONCLUSIONS}

The problem of estimating the threshold of a psychometric function has been extensively researched in the psychophysics literature, and practical applications in medical diagnosis have been found. We have reviewed existing algorithms for finding the threshold of a psychometric function, focusing on Bayesian adaptive methods and loss functions. We clearly established that for each (standard) loss function, there is a specific method of determining the final estimate that is most appropriate.

Six existing Bayesian methods of query selection were evaluated in this article, using a number of different loss functions. Previous results and intuition suggested the strategy of minimizing the expected loss of the posterior distribution for a few queries at the end of the estimation procedure, after minimizing the entropy for all previous queries. This new ENTFIRST scheme performed very well on a variety of simulations and tests. In particular, the problem of inaccurate estimates for the prior distribution, response function, and lapse and guess rates was consid- ered; ENTFIRST proved to be a more robust strategy than existing methods, even in these circumstances.

From a theoretical point of view, one important question for future research is to extend Paninski's (2005) work to provide a mathematical justification for why minimizing the posterior entropy gives better long-term results than minimizing the expected loss. This would also provide a mathematical justification for our algorithm, ENTFIRST, performing better than both the MinENT and MinLoss approaches to query selection.

From a practical point of view, the results in this study are limited to simulations using ideal prior, entropy, and loss functions that are used throughout the previous literature. There is some evidence that these carry over into practice, at least for visual field testing (Turpin, McKendrick, Johnson, \& Vingrys, 2002b). Although EnTFIRST is an effective threshold estimation procedure in these circumstances, we look forward to experiments that would confirm its role as a component in clinical psychophysical testing.

\section{AUTHOR NOTE}

E.K. is now with OMC International; J.M. is now with Robert Bosch Australia; A.T. is now with the University of Melbourne. Correspondence concerning this article should be addressed to A. Wirth, Department of Computer Science and Software Engineering, University of Melbourne, VIC 3010, Australia (e-mail: awirth@unimelb.edu.au).

\section{REFERENCES}

Alcalá-Quintana, R., \& García-Pérez, M. A. (2004). The role of parametric assumptions in adaptive Bayesian estimation. Psychological Methods, 9, 250-270. doi:10.1037/1082-989X.9.2.250

Bengtsson, B., \& Heisl, A. (1998). Evaluation of a new perimetric threshold strategy, SITA, in patients with manifest and suspect glaucoma. Acta Ophthalmologica Scandinavica, 76, 268-272 doi:10.1034/ j.1600-0420.1998.760303.x

Eilers, R. E., Ozdamar, O., \& Steffens, M. L. (1993). Classification of audiograms by sequential testing: Reliability and validity of an automated behavioral hearing screening algorithm. Journal of the American Academy of Audiology, 4, 172-181. doi:10.1121/1.400114

Hastie, T., Tibshirani, R., \& Friedman, J. (2001). The elements of statistical learning: Data mining, inference, and prediction. New York: Springer.

Henson, D. B., Chaudry, S., Artes, P. H., Faragher, E. B., \& Ansons, A. (2000). Response variability in the visual field: Comparison of optic neuritis, glaucoma, ocular hypertension, and normal eyes. Investigative Ophthalmology \& Visual Science, 41, 417-421.

HiLL, N. J. (2001). Testing hypotheses about psychometric functions. Unpublished doctoral dissertation, University of Oxford.

King-Smith, P. E., Grigsby, S. S., Vingrys, A. J., Benes, S. C., \& Supowit, A. (1994). Efficient and unbiased modifications of the QUEST threshold method: Theory, simulations, experimental evalu- 
ation and practical implementation. Vision Research, 34, 885-912. doi:10.1016/0042-6989(94)90039-6

KLEIN, S. A. (2001). Measuring, estimating, and understanding the psychometric function: A Commentary. Perception \& Psychophysics, 63, 1421-1455.

Kontsevich, L. L., \& Tyler, C. W. (1999). Bayesian adaptive estimation of psychometric slope and threshold. Vision Research, 39, 27292737. doi:10.1016/S0042-6989(98)00285-5

Kuss, M., JäKel, F., \& WichmanN, F. A. (2005). Bayesian inference for psychometric functions. Journal of Vision, 5, 478-492. doi:10 $.1167 / 5.5 .8$

Linschoten, M. R., Harvey, L. O., Jr., Eller, P. M., \& JafeK, B. W. (2001). Fast and accurate measurement of taste and smell thresholds using a maximum-likelihood adaptive staircase procedure. Perception \& Psychophysics, 63, 1330-1347.

PANINSKI, L. (2005). Asymptotic theory of information-theoretic experimental design. Neural Computation, 17, 1480-1507. doi:10.1162/ 0899766053723032

Ryan, A. (1976). Hearing sensitivity of the Mongolian gerbil, Meriones unguiculatis. Journal of the Acoustical Society of America, 59, 12221226. doi:10.1121/1.380961

TAYlor, M. M., \& CReElman, C. D. (1967). PEST: Efficient estimates on probability functions. Journal of the Acoustical Society of America, 41, 782-787. doi:10.1121/1.1910407

Treutwein, B. (1995). Adaptive psychophysical procedures. Vision Research, 35, 2503-2522. doi:10.1016/0042-6989(95)00016-S

Turpin, A., McKendrick, A. M., Johnson, C. A., \& Vingrys, A. J. (2002a). Development of efficient threshold strategies for frequency doubling technology perimetry using computer simulation. Investigative Ophthalmology \& Visual Science, 43, 322-331.

Turpin, A., McKendrick, A. M., Johnson, C. A., \& Vingrys, A. J. (2002b). Performance of efficient test procedures for frequency doubling technology perimetry in normal and glaucomatous eyes. Investigative Ophthalmology \& Visual Science, 43, 709-715.

Tyrell, R. A., \& Owens, D. A. (1988). A rapid technique to assess the resting states of the eyes and other threshold phenomena: The modified binary search (MOBS). Behavior Research Methods, Instruments, \& Computers, 20, 137-141.

Vingrys, A. J., \& Pianta, M. J. (1999). A new look at threshold estimation algorithms for automated static perimetry. Optometry \& Vision Science, 76, 588-595.

Watson, A. B., \& Pelli, D. G. (1983). QUEST: A Bayesian adaptive psychometric method. Perception \& Psychophysics, 33, 113-120.

Wichmann, F. A., \& Hill, N. J., (2001a). The psychometric function: I. Fitting, sampling, and goodness of fit. Perception \& Psychophysics, 63, 1293-1313.

WichmanN, F. A., \& Hill, N. J. (2001b). The psychometric function: II. Bootstrap-based confidence intervals and sampling. Perception \& Psychophysics, 63, 1314-1329.

WiLCOXON, F. (1945). Individual comparisons by ranking methods. Biometrics, 1, 80-83. doi:10.2307/3001968

(Manuscript received February 9, 2009; revision accepted for publication April 26, 2010.) 